\title{
Digitalisierung und Gesundheitswissenschaften - White Paper Digital Public Health
}

\section{Digitalization and Health Sciences - White Paper Digital Public Health}

(ㅇ) (1) $(8)$

Autoren

Ingrid Darmann-Finck ${ }^{1,3}$, Heinz Rothgang ${ }^{2,3}$, Hajo Zeeb ${ }^{3,4}$

Institute

1 Institut für Public Health und Pflegewissenschaft, Universität Bremen, Bremen

2 SOCIUM Forschungszentrum Ungleichheit und Sozialpolitik, Universität Bremen, Bremen

3 Wissenschaftsschwerpunkt Gesundheitswissenschaften, Universität Bremen, Bremen

4 Abt. Prävention und Evaluation, Leibniz-Institut für Präventionsforschung und Epidemiologie-BIPS, Bremen

\section{Schlüsselwörter}

Digital Public Health, Gerechtigkeit, nicht-intendierte

Effekte, Prävention, Gesundheitsförderung

\section{Key words}

Digital Public Health, Equity, non-intended effects, prevention, health promotion

online-publikation 2020

\section{Bibliografie}

Gesundheitswesen 2020; 82: 620-622

DOI 10.1055/a-1191-4344

ISSN $0941-3790$

(C) 2020. The Author(s).

This is an open access article published by Thieme under the terms of the Creative Commons Attribution-NonDerivative-NonCommercial-License, permitting copying and reproduction so long as the original work is given appropriate credit. Contents may not be used for commercial purposes, or adapted, remixed, transformed or built upon. (https://creativecommons. org/licenses/by-nc-nd/4.0/)

Georg Thieme Verlag KG, Rüdigerstraße 14,

70469 Stuttgart, Germany
Korrespondenzadresse

Prof. Hajo Zeeb

Abt. Prävention und Evaluation

Leibniz-Institut für Präventionsforschung und

Epidemiologie-BIPS

Achterstraße 30

28359 Bremen

zeeb@leibniz-bips.de

\section{ZUSAMMENFASSUNG}

Potenziale, Grenzen und Risiken der Digitalisierung in Public Health werden in den kommenden Jahren ein Schwerpunkt der Arbeit des Wissenschaftsschwerpunkts Gesundheitswissenschaften der Universität Bremen sein. Als grundlegende Positionsbestimmung in diesem dynamischen Themenfeld wurde ein White Papier entwickelt. Das White Paper benennt aus Sicht des Wissenschaftsschwerpunktes grundlegende Bewertungskriterien für Digital Public Health, identifiziert Schnittstellen und Ansatzpunkte der interdisziplinären Zusammenarbeit und beschreibt Querverbindungen und Abgrenzungen zu Themen der Digitalisierung in der Individualmedizin (Digital Health). Das White Paper wird hier in einer Kurzfassung zur Diskussion gestellt.

\section{ABSTRACT}

Potential limits and risk of digitalization in public health will be a core topic of the High profile Research Area Health Sciences at the University of Bremen in coming years. A white paper was developed to support positioning in this dynamic research. The paper describes the Health Sciences viewpoint on core evaluation criteria for digital public health, identifies interfaces and approaches for interdisciplinary cooperation and discusses cross-cutting themes as well as demarcations with respect to digitalization in medicine (digital health). An abbreviated version of the white paper is presented for discussion.

\section{Einleitung}

Der Wissenschaftsschwerpunkt Gesundheitswissenschaften ist einer von 6 Wissenschaftsschwerpunkten (WSP) der Universität
Bremen. Das übergeordnete Ziel des WSP Gesundheitswissenschaften besteht darin, mit gezielter Forschung zu einem genaueren Verständnis von Gesundheit, Krankheit und gesundheitlicher Versor- 
gung beizutragen und damit wissenschaftliche Grundlagen bereitzustellen, um die Gesundheit der Bevölkerung und die Qualität der Versorgung zu verbessern. Ein Schwerpunkt des WSP in den nächsten Jahren wird darin liegen, die Potenziale, Grenzen und Risiken der Digitalisierung in Public Health zu untersuchen. Das vorliegende White Papier wurde als grundlegende Positionsbestimmung in diesem dynamischen Themenfeld entwickelt. Zudem sollen Schnittstellen und Ansatzpunkte der interdisziplinären Zusammenarbeit identifiziert und Querverbindungen und Abgrenzungen zu Themen der Digitalisierung in der Individualmedizin (Digital Health) aufgezeigt werden.

Das zentrale Anliegen von Public Health und damit auch des Wissenschaftsschwerpunkts ist es, Nutzen und Effizienz gesundheitsbezogener Interventionen zu erforschen und zu verbessern und dabei Selbstbestimmung und Beteiligung der Bevölkerung sowie Verminderung von gesundheitlicher und sozialer Ungleichheit zu thematisieren. Ausgehend hiervon werden im Folgenden zunächst Bewertungskriterien von digitalen Technologien konkretisiert, die im Sinne und im Kontext von Public Health eingesetzt werden. Mit Bezug auf diese Kriterien werden im Anschluss Forschungsthemen und -bedarfe für die Ursachenforschung, Gesundheitsförderung und Prävention sowie Versorgung und Pflege abgeleitet.

\section{Bewertungskriterien für Digital Public Health}

Das zentrale Ziel von Digital Public Health ist es, die Verbesserung der Gesundheit der Bevölkerung durch die Anwendung neuer Technologien auf individueller, Community- und globaler Ebene voranzutreiben. Vor dem Hintergrund seiner Grundsätze stellt der WSP folgende übergeordnete Kriterien zur Bewertung derartiger Technologien in den Mittelpunkt der Forschung zu Digital Public Health.

\section{Effektivität und Effizienz}

Gesundheitsbezogene, digital unterstützte Public Health Interventionen sollten sich auf die besten verfügbaren wissenschaftlichen Erkenntnisse stützen, damit sie zum Erreichen der Public HealthZiele beitragen. Der WSP macht es sich zur Aufgabe, die Evidenzbasis hinsichtlich des Nutzens digitaler Technologien im Public Health Kontext zu untersuchen und in Bezug zu den verwendeten Ressourcen zu setzen, um so auch Aussagen zur Effizienz abzuleiten.

\section{Gerechtigkeit}

Gesundheitliche Gerechtigkeit ist ein zentrales Querschnittsthema der Gesundheitsforschung in Bremen. Gesundheitsbezogene Technologien müssen sich - den Nachweis des Nutzens vorausgesetzt - auch daran messen lassen, welche Auswirkungen sie auf gesundheitliche Ungleichheit und Gerechtigkeit haben. In den Forschungsprojekten des WSP werden diese Auswirkungen untersucht und ggf. Bedingungen ermittelt, mit denen beim Einsatz digitaler Technologien verhindert werden kann, dass sich bestehende gesundheitliche Ungleichheiten durch unterschiedlichen Zugang zu und Expertise im Umgang mit entsprechender Technik zwischen sozialen Gruppen vergrößern.

\section{Nicht-intendierte Effekte}

Auch digitale Anwendungen sollen nicht nur daran bemessen werden, ob sie effektiv und effizient sind und ob für beides hinreichende
Nachweise vorliegen. Vielmehr ist jeweils auch zu betrachten, ob und wenn ja, welche nicht-intendierten negativen und ggf. auch positiven Effekte ausgelöst werden. Im Zusammenhang mit Digital Public Health ist bspw. zu prüfen, ob möglicherweise eine Zunahme an Fremdbestimmung und ein gesellschaftlicher Druck zur gesundheitsschädlichen Selbstoptimierung oder auch ein Verlust unmittelbarer persönlicher oder gruppenbezogener Zuwendung und Interaktion festgestellt und wie diese unerwünschten Effekte vermieden werden können. Dabei stellt sich mit Blick auf den Klimawandel und den durch digitale Technologien verursachten Ressourcenverbrauch auch die Frage nach ökologischen Dimensionen der Bewertung.

\section{Forschungsthemen und -bedarf}

\section{Digitalisierung und gesundheitsbezogene Ursachenforschung}

Digitale Technologien werden vermehrt in der digitalen Epidemiologie eingesetzt und unterstützen die Datenerhebung z. B. durch mobile Eingabe von Daten oder durch Zuordnung von raumbezogenen Umweltdaten zu Individuen und Gruppen. Sie gewinnen täglich an Bedeutung in der Verknüpfung unterschiedlicher Datenquellen und der Analyse sehr großer, oft von Personen mittels ihrer Mobiltelefone generierter gesundheitsbezogener Daten, die häufig nicht primär für epidemiologische Studien erhoben wurden [1]. Für die Zukunft ist abzusehen, dass digitale Technologien auch die epidemiologische Ursachenforschung für nichtübertragbare Erkrankungen prägen werden.

Neben Chancen bergen diese Entwicklungen auch Risiken, insbesondere das Risiko, dass sich soziale Ungleichheiten in der Generierung von mobilen Daten (z. B. durch schlechtere technologische Ausstattung von sozioökonomisch benachteiligten Personen) verfestigen. Möglich ist auch, dass wichtige Risikofaktoren, die sich mit digitalen Ansätzen weniger gut erfassen oder messen lassen, auch weniger Beachtung erfahren. So gelingt es bisher besser, medizinnahe oder von Individuen selbst ermittelte Lebensstilrisikofaktoren mit digitalen Ansätzen zu erfassen als soziale Determinanten von Gesundheit und Krankheit. Mögliche Fehlentwicklungen durch entsprechende Verzerrungen müssen frühzeitig erkannt und korrigiert werden.

\section{Digitalisierung und Prävention/ Gesundheitsförderung}

Für Prävention und Gesundheitsförderung bieten digitale Technologien Möglichkeiten der breiten Dissemination von Maßnahmen bei vergleichsweise geringen Kosten. Typische Bereiche sind die Förderung körperlicher Aktivität und gesunder Ernährung bzw. die Gesundheitskommunikation in Bezug auf Impfungen oder Maßnahmen der Sekundärprävention. Da durch digitale Technologien Daten in Echtzeit auch über die Auswirkungen der Technologien auf die Nutzer/innen erhoben und ausgewertet werden können, ergeben sich außerdem neue Möglichkeiten der Nutzenmessung für Patient/innen und deren Versorgung. Einschränkend ist allerdings in Rechnung zu stellen, dass viele der App-basierten Angebote stark verhaltens- und nur wenig verhältnisorientiert sind. AuBerdem sollen Auswirkungen der Unterschiede in der digitalen Gesundheitskompetenz [2] ebenso überprüft werden wie der bisher 
vorliegende Befund der geringen längerfristigen Wirksamkeit von Digital Public Health Interventionen. Die Evidenzbasis für webbasierte und mobile Public Health-Maßnahmen der Prävention und Gesundheitsförderung ist insgesamt noch gering. Die hohe technologische Entwicklungsdynamik erschwert umfassende Evaluationen und erfordert neue Vorgehensweisen, da bisher genutzte Methoden angesichts der Schnelllebigkeit der Entwicklungen womöglich wenig geeignet sind.

\section{Digitalisierung und Versorgungsforschung/Pflege}

In den Bereichen gesundheitlicher Versorgung können 3 Schwerpunkte des Einsatzes digitaler Technologien unterschieden werden: Informations- und Kommunikationstechnologien, intelligente und vernetzte Robotik sowie vernetzte Hilfs- und Monitoringsysteme [3]. Während Informations- und Kommunikationstechnologien (z. B. elektronische Patientendokumentation, Krankenhausinformationssysteme) sowie die Überwachung von Vitalparametern durch Monitoringsysteme schon vergleichsweise weit verbreitet sind, befinden sich andere Anwendungsgebiete digitaler Technologien, etwa altersgerechte Assistenzsysteme für ein selbstbestimmtes Leben (AAL) oder Roboter, noch in der Erprobung [4].

Auch wenn die Potenziale der Digitalisierung zur Optimierung von Versorgungsabläufen stellenweise erkennbar sind, manifestieren sich Grenzen - neben fehlender Nutzennachweise - v. a. in Implementationsblockaden, die darauf zurückzuführen sind, dass die Entwicklung digitaler Unterstützungsangebote derzeit v. a. technologiegetrieben und nicht hinreichend auf die vorhandenen Bedarfe und Bedürfnisse sowohl von (älteren) versorgungsbedürftigen Menschen als auch von Akteur/innen der gesundheitlichen Versorgung abgestimmt ist. Insoweit Robotik menschliche Interaktion ersetzen soll, eröffnet sich zudem ein Spannungsfeld zwischen zunehmend individualisierender Technologieorientierung und direktem persönlichen Austausch sowie dem gemeinschaftlichen gesundheitsbezogenen Engagement auf lokaler, regionaler und globaler Ebene. Auch Risiken in Bezug auf Vereinzelung und Vereinsamung und Vergrößerung sozialer Ungleichheiten sind deutlich erkennbar.

\section{Methodologische und methodische Herausforderungen}

Neben Forschungsfragen hat der WSP in der Diskussion von Potenzialen und Grenzen von Digital Public Health einige zentrale, die Methodologie und die Methoden betreffende Leitsätze identifiziert:

- Ein transparentes, handlungsleitendes Rahmenkonzept mit gemeinsamen Definitionen von Konzepten und Begrifflichkeiten zu Digital Public Health ist für den von der interdisziplinären Public Health Forschungsgemeinde aktiv vorangetriebenen Erkenntnisgewinn und die Entwicklung des Gebietes von großer Bedeutung.

- Auf eine Evidenzbasierung als Basis eines Einsatzes entsprechender Technologien in Public Health kann nicht verzichtet werden, auch wenn die Entwicklung von Interventionsansätzen äußerst schnelllebig ist.

- Es sind Konzepte zu entwickeln, die angemessene Datensicherheit und höchsten Datenschutz insbesondere bei breitgefächerten, viele Menschen einbeziehenden Digital Public Health Anwendungen gewährleisten können.
- Digital Public Health sollte gemeinsam mit Bürgerinnen und Bürgern erforscht werden. Die partizipative Forschung ist nach dem Verständnis des WSP die Voraussetzung dafür, im Bereich Digital Public Health gültige und relevante Antworten auf Public Health-Forschungsfragen zu finden.

\section{Ausblick, Strukturen und Forschungsleitgedanken}

Der WSP Gesundheitswissenschaften räumt Digital Public Health einen wichtigen Platz in seiner Forschung ein. Um die Forschung auf diesem Gebiet zu intensivieren, hat der WSP mit dem Leibniz WissenschaftsCampus Digital Public Health Bremen (LWC DiPH) in Kooperation mit vielen inner- und außeruniversitären Mitwirkenden eine innovative und flexible Forschungsplattform geschaffen, die sich in 4 Forschungsclustern mit einer breiten Spanne von Aspekten von Digital Public Health beschäftigt.

Zusammenfassend bestehen die Ziele der international ausgerichteten Bremer Forschung zu Digital Public Health darin, die offensichtlichen Potenziale für Public Health genauer zu erforschen und zu optimieren. Dabei soll eine transparente und interdisziplinär angelegte Analyse und Bewertung von Risiken hohen Stellenwert erhalten. Mit Digital Public Health verbundene Risiken sollen tiefergehend untersucht und Konzepte zur Reduzierung der Risiken entwickelt und evaluiert werden. Diese Forschungsaktivitäten orientieren sich an den übergeordneten Grundsätzen von Public Health, wonach die Analyse von Ursachen und die darauf aufbauende Verbesserung der Gesundheit unter Berücksichtigung von Evidenz, Gerechtigkeit und vorhandenen Ressourcen angestrebt werden.

Eine Langversion dieses White Paper findet sich unter http:// www.healthsciences.uni-bremen.de/

Interessenkonflikt

Die Autorinnen/Autoren geben an, dass kein Interessenkonflikt besteht.

Literatur

[1] Park H-A, Jung H, On J et al. Digital epidemiology: Use of digital data collected for non-epidemiological purposes in epidemiological studies. Healthcare Informatics Research 2018; 24: 253. doi: 10.4258/ hir.2018.24.4.253

[2] Bittlingmayer UH, Dadaczynski K, Sahrai D et al. Digitale Gesundheitskompetenz - Konzeptionelle Verortung, Erfassung und Förderung mit Fokus auf Kinder und Jugendliche. Bundesgesundheitsblatt Gesundheitsforschung Gesundheitsschutz 2020; 63: 176-184. doi: 10.1007/ s00103-019-03087-6

[3] DAA Stiftung Bildung und Beruf Digitalisierung und Technisierung der Pflege in Deutschland. Aktuelle Trends und ihre Folgewirkungen auf Arbeitsorganisation, Beschäftigung und Qualifizierung. Hamburg: 2017; Online: https://www.daa-stiftung.de/fileadmin/user_upload/ digitalisierung_und_technisierung_der_pflege_2.pdf

[4] Krick T, Huter K, Domhoff D et al. Digital technology and nursing care: A scoping review on acceptance, effectiveness and efficiency studies of informal and formal care technologies. BMC Health Services Research 2019; 19: 400. doi:10.1186/s12913-019-4238-3 